\title{
High Dose IL-17A Enhances the Antiosteoarthritic Effect of Dental Pulp Mesenchymal Stem Cells by Reducing Inflammatory Response and Promoting Chondrocyte Proliferation
}

Fu Bi qi

First Affiliated Hospital of Nanchang University

Zhou Yao

First Affiliated Hospital of Nanchang University

Long Wei

First Affiliated Hospital of Nanchang University

Cai Fen

First Affiliated Hospital of Nanchang University

\section{Xia Qiang}

First Affiliated Hospital of Nanchang University

\section{Liu Ren}

First Affiliated Hospital of Nanchang University

Wu Rui ( $\nabla$ tcmclinic@163.com )

First Affiliated Hospital of Nanchang University https://orcid.org/0000-0003-0110-4912

\section{Research Article}

Keywords: Dental pulp mesenchymal stem cells, osteoarthritis, IL-17A, chondrocyte, inflammatory response

Posted Date: May 13th, 2021

DOI: https://doi.org/10.21203/rs.3.rs-471530/v1

License: (c) (1) This work is licensed under a Creative Commons Attribution 4.0 International License. Read Full License 


\section{Abstract}

Objective: Mesenchymal stem cells (MSCs) have promising potential in regenerative therapy, partially through their potent immune modulation. The inflammatory cytokine IL-17A has been suggested to improve the therapeutic efficacy of MSCs. Herein, we determined whether pretreatment of dental pulp MSCs (DPMSCs) with IL-17A enhances the therapeutic potency of DPMSCs for osteoarthritis (OA).

Methods: DPMSCs pretreated with IL-17A $(0-75 \mathrm{ng} / \mathrm{ml})$ were co-cultured with T lymphocytes for cytokine assay. In a rat OA model, DPMSCs pretreated with IL-17A were evaluated for antiosteoarthritic activity. Rat knee joints were harvested for color Doppler ultrasonography and histological staining, and synovial fluid was collected for cytokine assay. Additionally, chondrocytes isolated form joint cartilage of OA rats were co-cultured with IL-17A-pretreated DPMSCs for cell proliferation assay.

Results: DPMSCs demonstrated a pluripotent capacity to differentiate into osteocytes, adipocytes, and chondrocytes. For in vitro co-culture, although DPMSCs pretreated with low dose of IL-17A $(25 \mathrm{ng} / \mathrm{ml})$ had inflammation-promoting effect, indicated by increased IL-2 and TNF-a secretion and decreased IL-4 release, high dose IL-17A $(75 \mathrm{ng} / \mathrm{ml})$ pretreatment enhanced the anti-inflammatory activity of DPMSCs. In vivo, high dose IL-17A-pretreated DPMSCs showed a stronger antiosteoarthritic activity than untreated DPMSCs, indicated by significantly reducing joint swelling, synovial fluid formation, the OARSI scores, and the production of inflammatory cytokines in synovial fluid. Besides, high dose IL-17A- pretreated DPMSCs enhanced the stimulatory activity of DPMSCs in chondrocyte proliferation in vitro.

Conclusion: high dose IL-17A enhances the immunosuppressive property of DPMSCs, which helps alleviate osteoarthritis by inhibiting inflammatory response and promoting chondrocyte proliferation in the affected joints.

\section{Introduction}

Osteoarthritis $(\mathrm{OA})$ is a chronic and progressive degenerative disease associated with articular cartilage erosion, synovial inflammation, and subchondral bone remodeling, and can cause joint damage and even functional disability ${ }^{[1]}$. The risk and predisposing factors for $\mathrm{OA}$ include aging, genetics, mechanical stress, obesity, basic anatomical abnormalities, and trauma ${ }^{[2]}$. Previous studies have found that proinflammatory cytokines can induce an imbalance in the synthesis and degradation of articular chondrocytes, extracellular matrix, and subchondral bone, which is the pathological basis for developing $O A{ }^{[3,4]}$. At present, different strategies are applied to manage $O A$ depending on its clinical presentation and severity, including nonpharmacological interventions such as health education and weight control [5], pharmacological therapies such as glucosamine, non-steroidal anti-inflammatory drugs, and intraarticular injection of hyaluronic acid ${ }^{[6,7]}$, and surgery such as osteectomy and joint replacement in case of sever cartilage destruction ${ }^{[8]}$. However, these available strategies just control OA symptoms and are ineffective to delay the progressive degeneration of articular cartilage. 
Implantation of mesenchymal stem cells (MSCs) appears to hold the promise to treat articular cartilage degeneration ${ }^{[9]}$. There are many advantages to using MSCs as regenerative agents, including high selfrenewal capacity, multilineage differentiation potential, successful genome editing and immune silencing, easy isolation, cultivation and expansion, diverse immunomodulatory activity, and educating immune cells with greater immunosuppressive and anti-inflammatory properties ${ }^{[10]}$. A solid body of evidence shows that bone marrow-derived MSCs (BMMSCs) play a role in cartilage repair by generating new chondrocytes and preventing chondrocyte apoptosis via producing various mediators such as cytokines, antioxidants, erythropoietin, and heme oxidase ${ }^{[11,12]}$. Dental pulp MSCs (DPMSCs) are a unique type of MSCs with promising application in cell therapy. Compared to BMMSCs, DPMSCs can be easily obtained without ethical issues, and they have greater proliferation and stability, higher ability for cartilage and bone differentiation, and easier immune silencing ${ }^{[13]}$.

Immunophenotypes and immunoregulatory functions of MSCs can be shaped by local microenvironments. An inflamed-microenvironment can induce an immunophenotypic switch of MSCs to become either pro-inflammatory MSC-1 or anti-inflammatory MSC-2 phenotype, thus playing a broad immunomodulatory role through direct cell-cell contact or secreted signaling molecules ${ }^{[14]}$. It has been shown that low levels of inflammatory cytokines can drive MSCs toward pro-inflammatory MSC-1 and recruit lymphocytes to the inflammatory site to have pro-inflammatory activity. However, high levels of pro-inflammatory factors can drive MSCs with anti-inflammatory MSC-2 phenotype to exert immunosuppressive effect ${ }^{[15]}$.

IL-17A is a Th17 cell-derived inflammatory cytokine that has been investigated to preactivate MSCs and enhance the therapeutic efficacy of MSCs by inducing their anti-inflammatory phenotype. Therefore, we hypothesized that intra-articular injection of DPMSCs preactivated with high dose of IL-17A has a better therapeutic effect on OA by inhibiting intra-articular inflammation and alleviating chondrocyte apoptosis, thus preventing $\mathrm{OA}$ deterioration and progression.

\section{Materials And Methods}

\section{Ethics statement}

The study was approved by the Ethics Committee of the First Affiliated Hospital of Nanchang University. All participants signed the informed consent documentation prior to specimen collection. All animal experimental manipulations were carried out in strict accordance with the guidelines for Animal Experiments published by the National Institutes of Health.

\section{Cell preparation}

DPMSCs were obtained from CAR-T Biotechnology (Shanghai, China) and grown at $37^{\circ} \mathrm{C}$ in a-MEM supplemented with amino acids, vitamins, inorganic salts, and ribonucleosides in a $5 \% \mathrm{CO}_{2}$ incubator. DPMSCs were seeded in tissue culture flasks at a density of $4 \times 10^{3}$ cells $/ \mathrm{cm}^{2}$ overnight and then 
replaced with fresh medium containing $0-75 \mathrm{ng} / \mathrm{ml}$ recombinant human IL-17A (Bai ao lai bo, Beijing,China). After IL-17A treatment for 5 days, the cytokine was removed and cells were rinsed with Hank's balanced salt solution and detached for experiments as indicated elsewhere.

Peripheral blood mononuclear cells (PBMC) in peripheral blood samples from healthy volunteers were isolated by Ficoll-Paque (TBD, China) density gradient centrifugation. T cells ( $>80$ purity) were enriched by using nylon wool fiber columns (company, city, country). Articular cartilage was harvested from the hip joints of Sprague-Dawley rats as described below and chondrocytes were isolated by digesting at $37^{\circ} \mathrm{C}$ in medium containing $0.2 \%$ collagenase. For transwell co-culture, $\mathrm{T}$ lymphocytes and chondrocyte were cultured onto the membrane of transwell insert and DPMSCs were cultured on the bottom of transwell cell culture system, then incubated for different hours at $37^{\circ} \mathrm{C}$.

\section{Characterization of DPMSCs}

The immunophenotypes of DPMSCs were determined by flow cytometry analysis. Cells were harvested via trypsinization and incubated with monoclonal antibodies against CD73, CD90, CD29, CD44, CD105, CD146, CD34 and CD45 or isotype controls at $4^{\circ} \mathrm{C}$ for $30 \mathrm{~min}$, then cell surface markers were analyzed using a BD flow cytometry.

\section{Osteogenic differentiation}

When DPMSCs reached $80 \%$ confluence, medium was removed and replaced with osteogenic induction medium. Cells continued incubation in the induction medium for 4 weeks with medium refreshed every 3 days. After the formation of mineralized nodules, silver nitrate staining was performed and mineralized nodules, indicative of osteogenic differentiation, were observed under an inverted microscope and photographed.

\section{Adipogenic differentiation}

To initiate adipogenic differentiation, DPMSCs at $80 \%$ confluency were cultured in adipogenic induction medium for 4 weeks and the induction medium was refreshed every 3 days. After the appearance of lipid droplets, cells were stained with $0.3 \%$ Oil Red $O$ solution and then observed under an inverted microscope and photographed.

\section{Chondrogenic differentiation}

For chondrogenic differentiation, chondrogenic induction medium was added after DPMSCs achieved $80 \%$ confluence. The induction medium was changed every 3 days. After 4 weeks of culture in the induction medium, chondrogenic cells were viewed using the toluidine blue staining method.

\section{Rat model of osteoarthritis}

A total of 26 healthy female Sprague-Dawley rats were purchased from the Department of Zoology, Jiangxi University of Traditional Chinese Medicine and maintained at $22-24^{\circ} \mathrm{C}$, with a $12-\mathrm{h} \mathrm{light} /$ dark 
cycle and ad libitum access to food and water. At 12 weeks of age, all rats were injected with $4 \%$ papain $(0.1 \mathrm{ml}$ each on days 1,4 and 7$)$ into the knee joint cavity for establishing an OA animal model. After the last injection of papain, the rats were monitored for another week for general health and behavior. Two $\mathrm{OA}$ rats were sacrificed for primary culture of chondrocytes. The remaining 24 rats were randomly divided into 3 groups, 8 each. Group 1 was a control group which was injected with $0.1 \mathrm{ml}$ saline into the joint cavity once a week for four weeks. Group 2 was injected with $0.1 \mathrm{ml}$ saline containing $1 \times 10^{6}$ DPMSCs into the joint cavity once a week for four weeks. Group 3 was injected with the same number of DPMSCs once a week for four weeks, but DPMSCs had been pretreated with $75 \mathrm{ng} / \mathrm{ml} \mathrm{IL-17A} \mathrm{for} 5$ days.

After DPMSCs treatment, the rats were sacrificed by xxx. The injured knee joints were harvested for ultrasonography and histopathology analysis of cartilage damage and blood was collected for determining inflammatory mediators.

\section{Color Doppler ultrasonography}

The knee joints were subjected to color Doppler ultrasonography for assessing synovial inflammation, joint effusion, and cartilage damage (surface roughness).

\section{Histopathology}

The rat joints were fixed in $10 \%$ buffered paraformaldehyde for $48 \mathrm{~h}$. Prior to paraffin embedding, decalcification was performed for $6 \mathrm{~h}$ using 10\% EDTA (pH7.4) ${ }^{[16]}$. After paraffin embedding, articular cartilage specimens were cut into $3-\mu \mathrm{m}$ thick sectios and stained with hematoxylin and eosin (HE). The International Society for the Study of Osteoarthritis (OARSI) scoring system was used to assess the severity of cartilage damage ${ }^{[17]}$.

\section{Chemiluminescence immunoassay (CLIA)}

Rat serum and cell culture supernatants were collected and stored at $-80^{\circ} \mathrm{C}$ until assayed. IL-2, IL-4, TNF$a$, and IL-1 $\beta$ levels were detected by CLIA according to the manufacturer's instructions.

\section{Cell proliferation assay}

Chondrocyte proliferation was assayed using the Cell Counting Kit-8 (CCK-8) kit (Keygen Biotech, Jiangshu, China) according to the manufacturer's instructions. Briefly, after 24,48 , and $120 \mathrm{~h}$ of coculture, the chambers were removed and the CCK8 working solution was added to $10 \%$ of the total volume of culture medium of each well and cells continued incubation at $37^{\circ} \mathrm{C}$ for $1-4 \mathrm{~h}$. The absorbance was quantified at the wavelength of $450 \mathrm{~nm}$.

\section{Statistical analysis}

All statistical analyses were performed by using SPSS 22.0 (IBM, Chicago, IL, USA). Data are expressed as the mean \pm standard deviation (SD). Unpaired Student's $t$ test was used to compare two groups. For 
more than two groups, we used one-way analysis of variance (ANOVA) for statistical comparison followed by Bonferroni's post-test. A p value $<0.05$ was considered statistically significant.

\section{Results}

\section{Characterization of DPMSCs}

We extracted DPMSCs from the deciduous teeth of children. Representative microscopic images of cultured DPMSCs are shown in Fig. Undifferentiated DPMSCs exhibited a characteristic spindle-shaped morphology. Then, flow cytometric analysis of cell-surface markers was carried out to authenticate DPMSCs. The results showed that cells had an immunophenotype similar to that of MSCs, with high expression of MSCs markers CD73, CD90, CD29, CD44, CD105, and CD146, but were negative for the hematopoietic stem cell surface marker CD34 and immune cell surface marker CD45 (Fig. 1). Next, we analyzed the differentiation capacity of DPMSCs and found that DPMSCs harbored the potential to differentiate into osteocytes, adipocytes, and chondrocytes, similar to that of MSCs (Fig. 2).

\section{Differential effects of DPMSCs pretreated with different doses of IL-17A on T cell immune response}

Since immune modulation is involved in the MSCs treatment and their immunophenotype is subject to inflammatory factors, we assessed the immunomodulatory effects of DPMSCs pretreated with IL-17A on co-cultured T cell immune response. DPMSCs pretreated with different doses of IL-17A (0 (UT-DPMSC), 25 (low DPMSC-17), 50 (medium DPMSC-17), or $75 \mathrm{ng} / \mathrm{ml}$ (high DPMSC-17)) for 5 days were co-cultured with $T$ cells for another 5 days, then the secreted levels of pro-inflammatory cytokines, IL-2 and TNF-a, and anti-inflammatory IL-4 from T cells were determined. The results showed that compared to Control groups (the mono-cultured T cells), UT-DPMSC significantly inhibited co-cultured T cells to secrete IL-2 and TNF-a, but significantly enhanced the release of IL-4. Furthermore, compared to the UT-DPMSC treatment, low DPMSC-17 significantly increased the secreted levels of IL-2 and TNF- $a$, but decreased IL-4 secretion. On the contrary, high DPMSC-17 significantly inhibited the secretion of IL-2 and TNF- $a$ but induced IL-4 secretion (Fig. 3). These results indicate that DPMSCs alone drive T cells toward an antiinflammatory phenotype that secretes higher levels of IL-4 and lower levels of IL-2 and TNF-a. Moreover, our results suggest that IL-17A preactivation at different doses differentially regulates the immunophenotype of DPMSCs; high dose of IL-17A pretreatment $(75 \mathrm{ng} / \mathrm{ml})$ enhances the phenotype of DPMSCs toward the anti-inflammatory DPMSC-2 while low dose of IL-17A pretreatment $(25 \mathrm{ng} / \mathrm{ml})$ induces DPMSCs toward the pro-inflammatory DPMSC-1. Therefore, in the following experiments, we determined the therapeutic effect of the anti-inflammatory DPMSC-2 on OA in vivo.

\section{Antiosteoarthritic effects of high-dose IL-17A-pretreated DPMSCs in vivo}

After papain induction for 1 week, rats displayed decreased movement and obvious swelling and tenderness appeared in the affected joints. After four weeks of intro-articular injection therapy, compared to the placebo-treated control joints, the joint swelling in rats treated with DPMSCs or IL-17A-pretreated DPMDCs subsided significantly and the affected joints presented with normal skin temperature and 
without tenderness. Color Doppler ultrasonography of the affected joints in the placebo-treated control rats showed obvious joint swelling and fluid accumulation as well as visible blood flow signal and osteophyte formation. Relatively, the affected joints in DPMSCs-treated rats presented with moderate swelling, moderate effusion, and moderate hypoecho on the joint surface, and did not show obvious blood flow signal. Moreover, cartilage damage in rats treated with IL-17A-preactivated DPMSCs was further improved, presenting with slight swelling, a small amount of fluid, slight hypoecho on the joint surface, and no obvious blood flow signal (Fig. 4A), suggesting that IL-17A pretreatment improves the antiosteoarthritic efficacy of DPMSCs in the rat model of OA.

HE staining confirmed the findings by color Doppler ultrasound. The affected joints in the placebo-treated control displayed cartilage calcification, extensive fragmentation, severe cartilage degeneration, synovial tissue hyperplasia, and giant osteophyte formation. In DPMSCs-treated rats, moderate degeneration of articular cartilage, hyperplasia of synovial tissue, incomplete tidal line, and multiple obvious fractured areas were seen and articular cartilage was not collapsed into the epiphysis. Furthermore, the affected joints in rats treated with IL-17A-preactivated DPMSCs, showed slight articular cartilage degeneration, small osteophytes formation, and slight local fragmentation of calcified cartilage (Fig. 4B). According to the OARSI scoring system, DPMSCs and IL-17A-pretreaed DPMSCs had significantly lower scores compared to the placebo-treated control $(\mathrm{P}<0.05)$, and IL-17A-pretreaed DPMSCs achieved a much lower score than DPMSCs (Fig. 4C)..

CLIA assay of TNF- $\alpha$ and IL-1 $\beta$ levels in the synovial fluid showed that the levels of both cytokines were significantly different among those three treatment groups, generally, the pacebo-treated control > DPMSCs $>$ IL-17A-pretreaed DPMSCs $(P<0.05)$, further demonstrating that DPMSCs are an immunomodulatory agent and high dose IL-17A pretreatment could further enhance the antiinflammatory potential of DPMSCs.

\section{High dose of IL-17A enhances the stimulatory effect of DPMSCs on chondrocyte proliferation in vitro}

To understand the mechanism behind the enhancement of the antiosteoarthritic potential of DPMSCs by high dose IL-17A, we comparatively determined the effects of DPMSCs and IL-17A-pretreated counterparts on chondrocyte proliferation. To do this, chondrocytes were isolated from articular cartilage of rats with OA and co-cultured with DPMSC or IL-17A (75 ng/ml)-pretreated DPMSC (1:1) in Transwells for 24,48 , and $120 \mathrm{~h}$, then chondrocyte proliferation was determined by the CCK 8 assay. The results showed that both DPMSCs and IL-17A-pretreated DPMSCs increased the proliferation ability of chondrocytes, and IL-17A-pretreated DPMSCs produced an enhancing effect on chondrocyte proliferation than the untreated DPMSCs (Fig. 5). These results suggest that DPMSCs pretreated with high dose of IL17A possess an enhancing capacity for promoting chondrocyte proliferation.

\section{Discussion}

$\mathrm{OA}$ is the most common inflammatory joint disease that causes joint pain and functional loss, currently affecting almost 240 million people worldwide. OA was estimated to be the fourth leading cause of 
disability globally in $2020^{[18]}$. Unfortunately, until now, little is known of the pathogenesis of OA. However, increasing evidence has indicated that the dysregulation of inflammatory mediators in the articular cavity play a pivotal role in the occurrence of $\mathrm{OA}$, leading to cartilage degeneration, synovial hyperplasia, and chondrocyte apoptosis ${ }^{[3,4]}$. Current $\mathrm{OA}$ treatments often cause side effects or require invasive surgery ${ }^{[5-}$ 8]. In recent years, MSCs-based regenerative therapy appears to hold promise for OA treatment because of their ability to differentiate into chondrocytes, which help repair damaged cartilage ${ }^{[9]}$. In addition, modified MSCs may evade immune clearance and regulate immune cells to achieve immunosuppressive and anti-inflammatory functions ${ }^{[19]}$.

As a specific type of MSCs isolated from human exfoliated deciduous teeth [20], DPMSCs with easy availability, weak immunogenicity and strong differentiation capacity has emerged as ideal stem cells for the treatment of $\mathrm{OA}^{[21]}$. To ensure the authenticity of DPMSCs used in this study, we first identified and validated their cell-surface antigen profile according to the ISCT standard. We also confirmed that these DPMSCs have adipogenic, osteogenic, and chondrogenic differentiation potentials in different external environments.

Previous studies have demonstrated that different inflammatory cues could regulate the immunophenotypic switch of MSCs to become either pro-inflammatory or anti-inflammatory $[14,15]$. Therefore, several cytokines including IL-17A have been assessed for their therapeutic importance in enhancing the efficacy of MSCs. Since its discovery, IL-17A has been thought to accelerate the progression of diverse array of autoimmune diseases ${ }^{[22,23]}$. IL-17A has been documented to hold the key to joint destruction because this inflammatory cytokine is not only present in the synovial fluid of OA patients but also its levels are positively correlated with disease activity ${ }^{[24]}$. Further studies demonstrated that IL-17A can enhance the expression of matrix metalloproteinase (MMP)-9, MMP-13, and other MMPs in chondrocytes, induce collagenases in the cartilage damage, strengthen cartilage plate destruction and cartilage tissue decomposition, and prevent chondrocyte regeneration and tissue repair ${ }^{[25.26]}$, indicating a key role of IL-17A in the pathogenesis of OA. On the other hand, IL-17A is considered a growth factor for MSCs that acts synergistically with IFN- $y$ and TNF-a to enhance MSCs-mediated immunosuppression [27]. Thus, in our study DPMSCs were pretreated with different doses of IL-17A and co-cultured with T lymphocytes to determine their immunomodulatory changes. We found that IL-17A at $75 \mathrm{ng} / \mathrm{ml}$ enhanced the immnosuppresive effects of DPMSCs on the secretion of proinflammatory cytokines by $T$ lymphocytes in vitro.

Swart et al. ${ }^{[28]}$ found that in a mouse model of OA, intraperitoneal injection of MSCs reduced the severity of cartilage and bone injury. Another study observed that intra-articular injection of MSCs ameliorated systemic inflammatory response ${ }^{[29]}$. In this study, we confirmed that the antiosteoarthritic effects of intra-articular injection of DPMSCs in OA rats through exerting anti-inflammatory and immunomodulatory action, and found that DPMSCs preactivated with high dose of IL-17A had better performance in improving cartilage damage and bone erosion. This observation was in accordance with previous findings of the enhanced efficacy of MSCs by IL17A ${ }^{[27]}$. 
To help understand the role of high dose IL-17A in promoting the antiosteoarthritic efficacy of DPMSCs in $O A$ rats, we isolated chondrocytes from OA rats and evaluated the regulation of the DPMSC effects on chondrocyte proliferation by high dose IL-17A. We found that DPMSCs stimulated chondrocyte proliferation and such enhanced cell proliferation by DPMSCs was further enhanced by the high dose IL17A pretreatment, suggesting the enhanced chondrocyte proliferation is one of the mechanisms for the improved efficacy of IL-17A-pretreated DPMSCs.

In conclusion, we for the first time demonstrated that high dose IL-17A could modify DPMSCs to harbor more robust immunosuppressive properties, which reduced the joint destruction by inhibiting inflammatory response and promoting chondrocyte proliferation in the $\mathrm{OA}$ rats.

\section{References}

1. David J Hunter, Sita Bierma-Zeinstra. Osteoarthritis.Lancet 2019; 393: 1745-1759.

2. Sacitharan Pradeep Kumar. Ageing and Osteoarthritis. Subcell Biochem, 2019, 91: 123-159.

3. van Meurs J B J, Uitterlinden A G. Osteoarthritis year 2012 in review: genetics and genomics. Osteoarthritis Cartilage, 2012, 20: 1470-6.

4. Liu-Bryan Ru, Terkeltaub Robert. Emerging regulators of the inflammatory process in osteoarthritis. Nat Rev Rheumatol, 2015, 11: 35-44.

5. Stürmer T, Günther K P, Brenner H. Obesity, overweight and patterns of osteoarthritis: the Ulm Osteoarthritis Study. J Clin Epidemiol, 2000, 53: 307-13.

6. Migliore Alberto, Paoletta Marco, Moretti Antimo et al. The perspectives of intra-articular therapy in the management of osteoarthritis. Expert Opin Drug Deliv, 2020, 17: 1213-1226.

7. Beaudart C, Lengelé L, Leclercq V et al. Symptomatic Efficacy of Pharmacological Treatments for Knee Osteoarthritis: A Systematic Review and a Network Meta-Analysis with a 6-Month Time Horizon. Drugs, 2020, 80: 1947-1959.

8. Makris Eleftherios $\mathrm{A}$, Gomoll Andreas $\mathrm{H}$, Malizos Konstantinos $\mathrm{N}$ et al. Repair and tissue engineering techniques for articular cartilage. Nat Rev Rheumatol, 2015, 11: 21-34.

9. Perdisa Francesco, Gostyńska Natalia, Roffı Alice et al. Adipose-Derived Mesenchymal Stem Cells for the Treatment of Articular Cartilage: A Systematic Review on Preclinical and Clinical Evidence. Stem Cells Int, 2015, 2015: 597652.

10. Kyurkchiev Dobroslav, Bochev Ivan, Ivanova-Todorova Ekaterina et al. Secretion of immunoregulatory cytokines by mesenchymal stem cells. World J Stem Cells, 2014, 6: 552-70.

11. Vonk Lucienne A, van Dooremalen Sanne F J, Liv Nalan et al. In Vitro Mesenchymal Stromal/stem Cell-derived Extracellular Vesicles Promote Human Cartilage Regeneration. Theranostics, 2018, 8: 906-920.

12. Diekman Brian O, Guilak Farshid. Stem cell-based therapies for osteoarthritis: challenges and opportunities. Curr Opin Rheumatol, 2013, 25: 119-26. 
13. Ponnaiyan Deepa, Jegadeesan Visakan. Comparison of phenotype and differentiation marker gene expression profiles in human dental pulp and bone marrow mesenchymal stem cells. Eur J Dent, 2014, 8: 307-313.

14. Waterman Ruth S, Tomchuck Suzanne L, Henkle Sarah L et al. A new mesenchymal stem cell (MSC) paradigm: polarization into a pro-inflammatory MSC1 or an Immunosuppressive MSC2 phenotype. PLoS One, 2010, 5: e10088.

15. Selmani Zohair, Naji Abderrahim, Zidi Ines et al. Human leukocyte antigen-G5 secretion by human mesenchymal stem cells is required to suppress $T$ lymphocyte and natural killer function and to induce CD4+CD25highFOXP3+ regulatory T cells. Stem Cells, 2008, 26: 212-22.

16. Min Zhang, Xiaomeng Liu, Zheng Li et al. Asymmetrical methyltransferase PRMT3 regulates human mesenchymal stem cell osteogenesis via miR-3648. Cell Death Dis, 2019, 10: 581.

17. Glasson S S, Chambers M G, Van Den Berg W B et al. The OARSI histopathology initiative recommendations for histological assessments of osteoarthritis in the mouse. Osteoarthritis Cartilage, 2010, S17-23.

18. Kim Yong Sang, Suh Dong Suk, Tak Dae Hyun et al. Mesenchymal Stem Cell Implantation in Knee Osteoarthritis: Midterm Outcomes and Survival Analysis in 467 Patients. Orthop J Sports Med, 2020, 8: 2325967120969189.

19. Le Blanc K, Ringdén O. Immunomodulation by mesenchymal stem cells and clinical experience. J Intern Med, 2007, 262: 509-25.

20. Yu Fan, Dong Yan, Yang Yan-Wei et al. Effect of an Experimental Direct Pulp-capping Material on the Properties and Osteogenic Differentiation of Human Dental Pulp Stem Cells. Sci Rep, 2016, 6: 34713.

21. Liu Junjun, Yu Fang, Sun Yao et al. Concise reviews: Characteristics and potential applications of human dental tissue-derived mesenchymal stem cells. Stem Cells, 2015, 33: 627-38.

22. Beringer Audrey, Miossec Pierre. Systemic effects of IL-17 in inflammatory arthritis. Nat Rev Rheumatol, 2019, 15: 491-501.

23. Na Hyun Sik, Park Jin-Sil, Cho Keun-Hyung et al. Interleukin-1-Interleukin-17 Signaling Axis Induces Cartilage Destruction and Promotes Experimental Osteoarthritis. Front Immunol, 2020, 11: 730.

24. Nowicka-Stążka Patrycja, Langner Ewa, Turski Waldemar et al. Quinaldic acid in synovial fluid of patients with rheumatoid arthritis and osteoarthritis and its effect on synoviocytes in vitro. Pharmacol Rep, 2018, 70: 277-283.

25. Deligne C, Casulli S, Pigenet A et al. Differential expression of interleukin-17 and interleukin-22 in inflamed and non-inflamed synovium from osteoarthritis patients. Osteoarthritis Cartilage, 2015, 23: 1843-52.

26. Woodell-May Jennifer, Matuska Andrea, Oyster Megan et al. Autologous protein solution inhibits MMP-13 production by IL-1 $\beta$ and TNFa-stimulated human articular chondrocytes. J Orthop Res, 2011, 29: 1320-6.

27. Song Ji-Young, Kang Hyo Jeong, Ju Hyun Min et al. Umbilical cord-derived mesenchymal stem cell extracts ameliorate atopic dermatitis in mice by reducing the T cell responses. Sci Rep, 2019, 9: 
6623.

28. Swart J F, de Roock S, Hofhuis F M et al. Mesenchymal stem cell therapy in proteoglycan induced arthritis. Ann Rheum Dis, 2015, 74: 769-77.

29. Chang Yu-Hsun, Wu Kun-Chi, Ding Dah-Ching. Induced Pluripotent Stem Cell-Differentiated Chondrocytes Repair Cartilage Defect in a Rabbit Osteoarthritis Model. Stem Cells Int, 2020, 2020: 8867349.

\section{Figures}

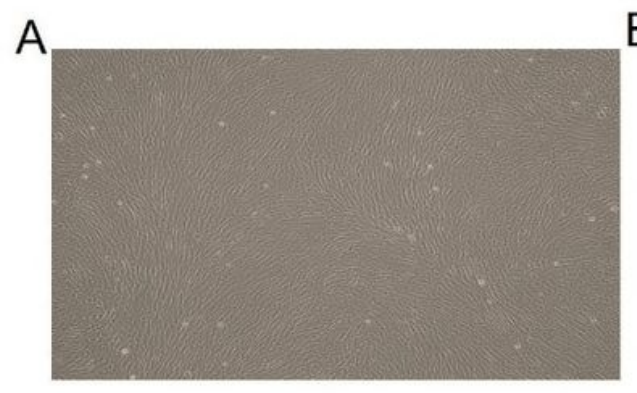

B
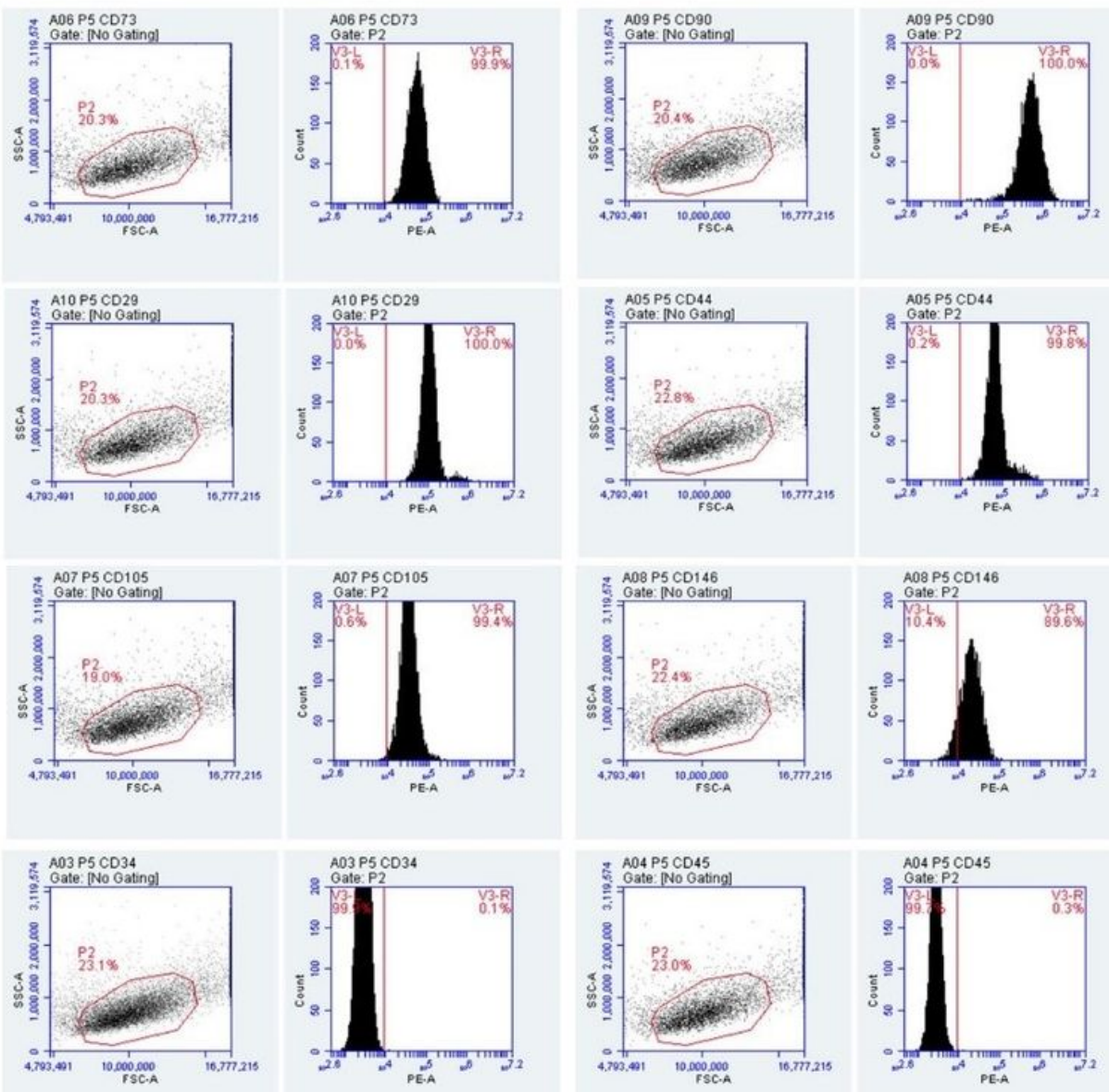

\section{Figure 1}

Successful isolation and phenotypic characterization of DPMSCs. A: Morphology of DPMSCs observed under an inverted microscope. B. The expression of surface markers for DPMSCs (CD73, CD90, CD29, CD44, CD105, CD146, CD34 and CD45) by flow cytometry. 
A

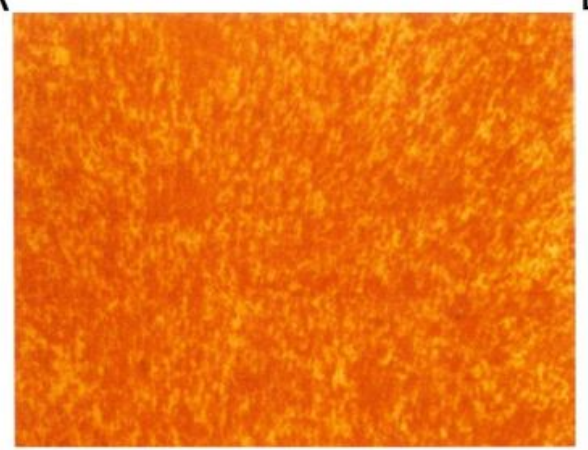

B

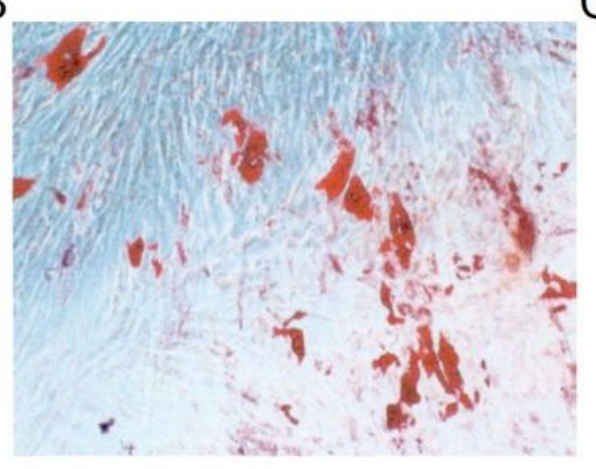

C

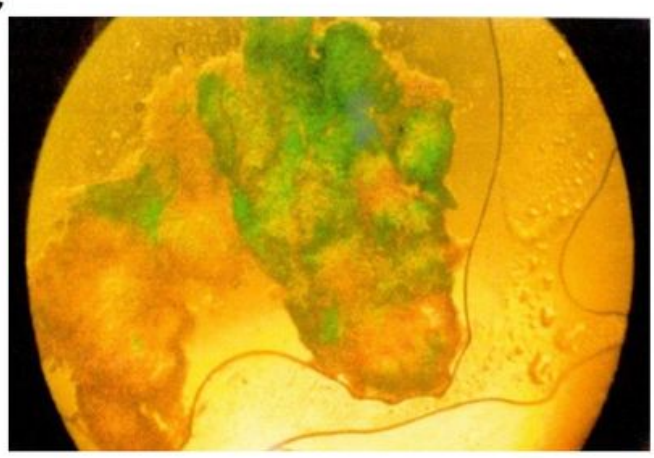

Figure 2

DPMSCs show the pluripotent differentiation capacity. A: Induction of adipogenic differentiation. B: Induction of osteogenic differention. C: Induction of chondrogenic differentiation.
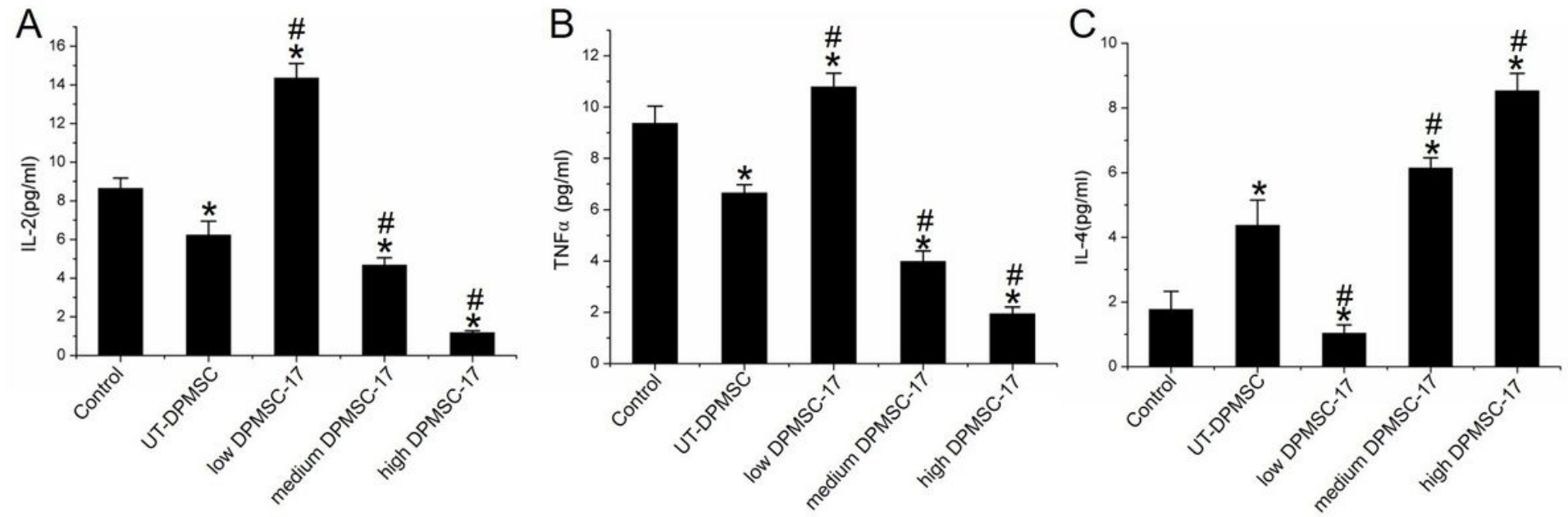

Figure 3

Effects of IL-17A-pretreated DPMSCs on T cell immune response. CLISA assay of inflammatory factors (IL-2 (A), TNF-a (B) and IL-4 (C)) in culture medium of DPMSCs pretreated with different doses of IL-17A $(0-75 \mathrm{ng} / \mathrm{ml})$ and co-cultured with $\mathrm{T}$ lymphocytes for 5 days. Data are presented as the mean $\pm \mathrm{SD}(\mathrm{n}=3)$. 

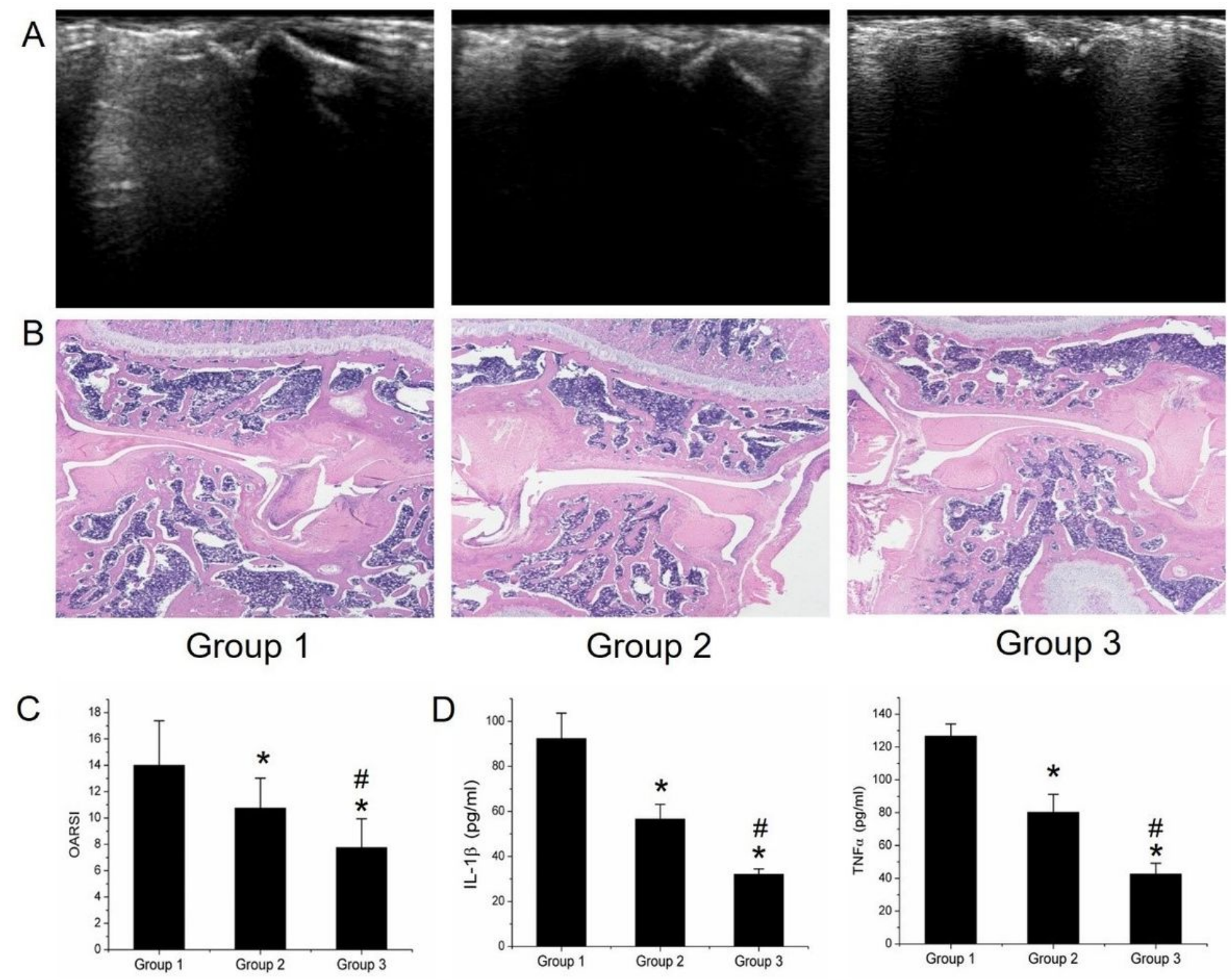

\section{Group 3}
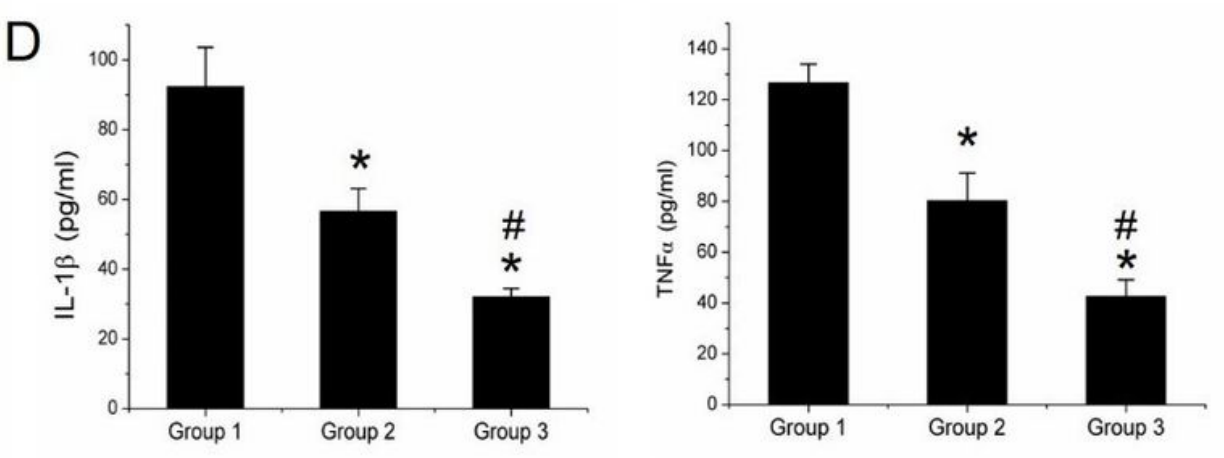

\section{Figure 4}

High dose IL-17A-pretreated DPMSCs alleviates joint injury and inflammation in OA rats. A: Joint structure observed by color Doppler ultrasonography. B: HE staining of joint cartilage. C: The OARSI scoring of joint cartilage. D: CLISA assay of the levels of inflammatory factors (IL-1 $\beta$ and TNF-a) in joint fluid. Data are expressed as the mean $\pm S D(n=8)$. 


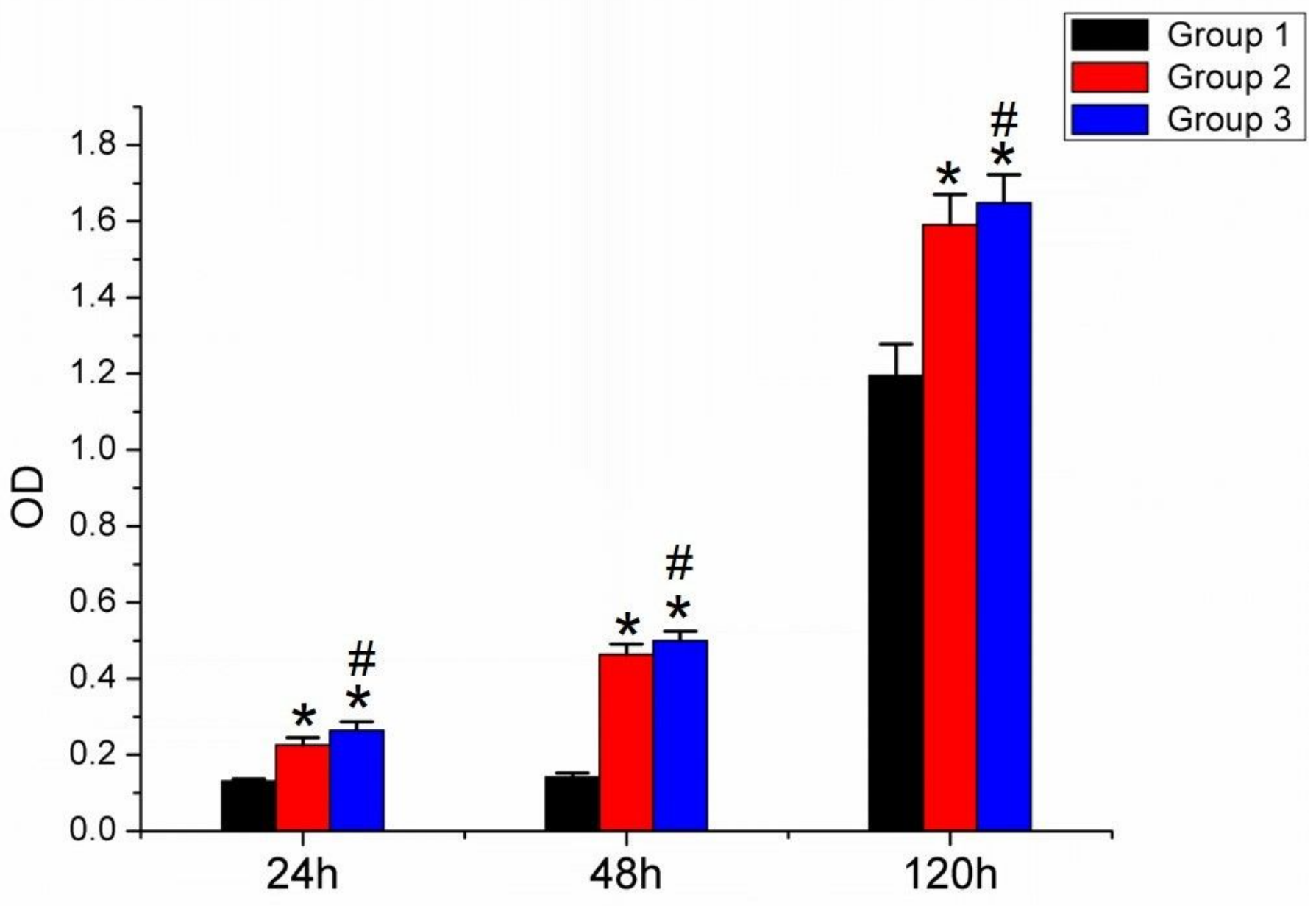

Figure 5

High dose IL17A-pretreated DPMSCs stimulates chondrocyte proliferation in vitro. Cell proliferation was analyzed by a colorimetric assay kit after co-cultured with DPMSCs after 24,48 , and $120 \mathrm{~h}$. Data are presented as the mean $\pm S D(n=3)$. 